\title{
A THEOREM OF NILPOTENT GROUPS
}

\section{CHONG-YUN CHAO}

According to Wiman in [4] and Blackburn in [1], a p-group $H$ is said to be of maximal class if the lower central series of $H$,

$$
H=H_{1} \supset H_{2} \supset \cdots \supset H_{t} \supset H_{t+1}=\{e\}, \quad t \geqq 2,
$$

has the property that $H / H_{2}$ is of order $p^{2}$, and $H_{i} / H_{i+1}$ is of order $p$, $i=2,3, \cdots, t$. Thus, the center of $H$ is $H_{t}$ whose order is $p$. It seems natural to ask whether one can have a nilpotent group $G$ whose $G_{i}$, $i \geqq 2$, is a $p$-group of maximal class where $G=G_{1} \supset G_{2} \supset \cdots \supset G_{r+1}$ $=\{e\}$ is the lower central series of $G$. From a theorem of Burnside, [2, p. 241], we know that $H$ cannot be imbedded as the commutator subgroup of a p-group. Also, from a result of Hobby in [3, Theorem $1]$, we know that $H$ cannot be the Frattini subgroup of a $p$-group. In fact, Remark 1 in [3] gives a more general result. Here we shall give a negative answer to our question by proving the following more general theorem:

Let $G$ be a nilpotent group. A subgroup $K$ of $G$ is said to be a $\Psi$ group of $G$ if $K$ is invariant in $G$ and $G_{2} \supseteq K \supseteq G_{r-1}$ where $G=G_{1} \supset G_{2}$ $\supset \cdots \supset G_{r-1} \supset G_{r} \supset G_{r+1}=\{e\}$ is the lower central series of $G$. Clearly, each $G_{i}, 2 \leqq i \leqq r-1$, is a $\Psi$-group of $G$.

Theorem. A nonabelian group whose center is either a cyclic group of order $p$ (a prime) or a cyclic group of infinite order cannot be a $\Psi$ group of a nilpotent group $G$.

Proof. Let $G=G_{1} \supset G_{2} \supset \cdots \supset G_{r-1} \supset G_{r} \supset G_{r+1}=\{e\}$ be the lower central series of $G, H$ be a nonabelian group and $Z(H)$ be the center of $H$ such that $Z(H)$ is either a cyclic group of order $p$ or a cyclic group of infinite order. Suppose the contrary, i.e. $H$ is an invariant subgroup of $G$ and $G_{2} \supseteq H \supseteq G_{r-1}$. Since $Z(H)$ is a characteristic subgroup of $H$ and $H$ is invariant in $G, Z(H)$ is an invariant subgroup of $G$. Hence, $g Z(H) g^{-1} \subseteq Z(H)$ for every $g \in G$. Let $x$ be a generator of $Z(H)$. Then $g x g^{-1}=x^{n+1}$. We claim that if $Z(H)$ is cyclic of order $p$ then $n \equiv 0$ $\bmod p$ for every $g \in G$, and that if $Z(H)$ is cyclic of infinite order then $n$ must be zero for every $g \in G$. Suppose there is a $g \in G$ such that $[g, x]=g x g^{-1} x^{-1}=x^{n} \neq e$. Assume

$$
\begin{gathered}
{\left[g,[g, \mid \cdots[g, x] \cdots]=x^{n^{m-1}},\right.} \\
(m-1 \text { terms })
\end{gathered}
$$

Received by the editors March 7, 1967, and, in revised form, April 20, 1967. 
then

$$
\begin{aligned}
& {[g,[g,[g,[\cdots[g, x] \cdots]} \\
& \text { ( } m \text { terms) } \\
& \begin{array}{l}
=\left(g x^{n^{m-1}} g^{-1}\right)\left(x^{n^{m-1}}\right)^{-1}=\left(g x g^{-1}\right)^{n^{m-1}}\left(x^{n^{m-1}}\right)^{-1} \\
=\left(x^{n+1}\right)^{n^{m-1}}\left(x^{n^{m-1}}\right)^{-1}=x^{n^{m}} .
\end{array}
\end{aligned}
$$

For the case of $Z(H)$ being a cyclic group of order $p, n \neq 0 \bmod p$ implies $x^{n} \neq e$ and $x^{n^{m}} \neq e$ for any positive integer $m$. For the case of $Z(H)$ being a cyclic group of infinite order, $n \neq 0$ implies $x^{n} \neq e$ and $x^{n^{m}} \neq e$ either for any positive integer $m$. Hence, in either case, the lower central series of $G$ will never reach identity. That is a contradiction to $G$ being nilpotent. Hence we have $g x g^{-1}=x$ for every $g \in G$, i.e. $x \in Z(G)$ and $Z(H) \subseteq Z(G)$.

Since $G / Z(H)$ is nilpotent, we have its lower central series:

$(G / Z(H))=\left(G_{1} / Z(H)\right) \supset\left(G_{2} / Z(H)\right) \supset \cdots \supset\left(\left(G_{r-1} Z(H)\right) / Z(H)\right)$ $\supset\left(\left(G_{r} Z(H)\right) / Z(H)\right) \supseteq\{\bar{e}\}$. Since $H$ is nonabelian and $H \supseteq G_{r-1}$, there is a nonidentity $\bar{y} \in(Z(G / Z(H)) \cap(H / Z(H)))$, i.e. if $\left(G_{r} Z(H) / Z(H)\right)$ $\neq\{\bar{e}\}$, then $\bar{y} \in\left(G_{r} Z(H) / Z(H)\right)$. If $\left(G_{r} Z(H) / Z(H)\right)=\{\bar{e}\}$ then $\bar{y} \in\left(G_{r-1} Z(H) / Z(H)\right)$. Since $[\bar{y}, \bar{u}]=\bar{e}$ for every $\bar{u} \in(G / Z(H))$, we have $y u y^{-1} u^{-1}=x^{n} y u y^{-1} u^{-1}=x^{n(y, u)}$ where $n(y, u)$ is an integer, $\bar{y}=y Z(H)$ and $\bar{u}=u Z(H)$.

Let $v$ be any element of $G$. Then, using the fact $x \in Z(G)$, we have

$$
\begin{aligned}
& {[y,[u, v]]=y\left(u v u^{-1} v^{-1}\right) y^{-1}\left(u v u^{-1} v^{-1}\right)^{-1}=y u v u^{-1}\left(v^{-1} y^{-1} v y\right) y^{-1} u v^{-1} u^{-1}} \\
& \quad=y u v\left(u^{-1} y^{-1} u y\right) y^{-1} v^{-1} u^{-1} x^{-n(y, v)}=y u\left(v y^{-1} v^{-1} y\right) y^{-1} u^{-1} x^{-n(y, u)} x^{-n(y, v)} \\
& \quad=x^{n(y, u)} x^{n(y, v)} x^{-n(y, u)} x^{-n(y, v)}=e .
\end{aligned}
$$

When $u$ and $v$ go through $G, y$ commutes with every generator of $G_{2}$. Hence, $y \in Z\left(G_{2}\right)$. In particular, $y$ commutes with every element in $H$ since $H \subseteq G_{2}$. This means $y \in Z(H)$. That is a contradiction. Hence, $H$ cannot be a $\Psi$-group of a nilpotent group $G$.

\section{REFERENCES}

1. N. Blackburn, On a special class of p-groups, Acta Math. 100 (1958), 45-92.

2. W. Burnside, On some properties of groups whose orders are powers of primes, Proc. London Math. Soc. (2) 11 (1912), 225-245.

3. C. Hobby, The Frattini subgroup of a p-group, Pacific J. Math. 10 (1960), 209212.

4. A. Wiman, Über p-Gruppen von maximaler Klasse, Acta Math. 88 (1952), 317-346.

University of Pittsburgh 